\title{
Actualización en pruebas de susceptibilidad antifúngica
}

\author{
Cecilia V. Tapia P.
}

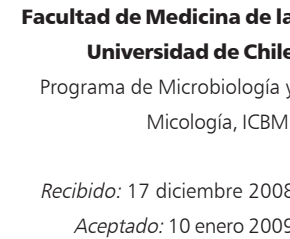

El autor declara no tener conflictos de interés relacionados a este

trabajo.

No existe fuente de financiamiento para la realización de esta revisión.

Correspondencia a: Cecilia V. Tapia Paredes cetapia@med.uchile.cl

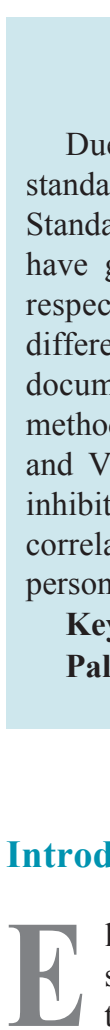

Due to increasing of invasive fungal infections and emergency of antifungal drugs resistant fungi, standardized methods of antifungal susceptibility testing (AST) have been developed. The Clinical Laboratory Standards Instutute (CLSI) and the European for Committee Antimicrobial Susceptibility Testing (EUCAST) have guidelines for susceptibility of yeasts by broth microdilution (M27-A2 and E. Dis. 7.1 documents, respectively). Both are equivalent, although they present methodological and interpretative breakpoints differences. In addition, the CLSI have the M38-A (for filamentous fungi) and M44-A (disk diffusion) documents, whereas EUCAST is developing a document for Aspergillus spp. Furthermore, commercial methods are available that display good correlation with the methods of reference such as E-test ${ }^{\circledR}$, Sensititre ${ }^{\mathbb{R}}$ and Vitek $2^{\circledR}$. The interpretation of the results must be careful because the determination of the minimum inhibitory concentration (CIM) is difficult for fungi, there are host factors involved and not always there is a correlation between MIC and clinical outcome. Due to these methods are laborious and require trained personnel, to ask for AST to a reference laboratory is recommendable.

Key words: Fungal drugs sensitivity tests, Candida, moulds, casts.

Palabras clave: Susceptibilidad antifúngica, Candida, hongos filamentosos, levaduras.

\section{Introducción} incremento en las infecciones fúngicas invasoras se ha mantenido constante en el ambiente hospitalario, debido fundamentalmente a un mayor número de pacientes con patologías de riesgo, tales como leucemias, cáncer con neutropenia induci$\mathrm{da}$, pacientes con trasplante de precursores hematopoyéticos sometidos a terapias inmunosupresoras y pacientes con SIDA ${ }^{1}$. Por otra parte, se ha descrito la aparición de resistencia secundaria en cepas de Candida expuestas a fármacos fungiestáticos como los azoles $^{2,3}$ y el aislamiento de especies intrínsecamente resistentes a determinados antifúngicos como C. krusei, y hongos filamentosos como Zigomycetes y Scedosporium prolificans ${ }^{4}$. Dado que estas infecciones se asocian a una alta mortalidad y que existe falla terapéutica relacionada al aislamiento de cepas resistentes es necesario contar con algún método para medir susceptibilidad antifúngica ${ }^{5}$

Los estudios de susceptibilidad a antifúngicos anteriores al uso de métodos estandarizados, eran inconsistentes y muy poco reproducibles, ya que hay muchos factores que influyen en estos ensayos, como el tamaño del inóculo, la composición y $\mathrm{pH}$ del medio, formato de la prueba y temperatura de incubación ${ }^{6,7}$. En 1992 apareció el primer estándar internacional para susceptibilidad de levaduras elaborado por el National Committee for Clinical Laboratory Standards (NCCLS), actualmente Clinical Laboratory Standards Institute (CLSI) en E.U.A., basado en un método de macrodilución en caldo (adaptado posteriormente a microdilución). Este documento (M27-A) fue aprobado en 1997 y permite medir las CIMs de las principales especies de levaduras oportunistas (Candida sp, Cryptococcus neoformans $)^{8}$ demostrando una adecuada reproducibilidad interlaboratorio en diversos estudios multicéntricos ${ }^{6,7}$.

Con mayor dificultad se ha podido establecer una metodología para la evaluar susceptibilidad de hongos filamentosos microscópicos. El CLSI cuenta con un estándar (documento M38-A) para algunos hongos fillamentosos ${ }^{9,11}$. Además, en los últimos años, se ha logrado estandarizar el método de difusión en agar en el documento M-44 del CLSI ${ }^{10,11}$.

Por otra parte, también se ha desarrollado un estándar europeo, Subcommittee on Antifungal Susceptibility Testing (AFST) del European Committee on Antimicrobial Susceptibility Testing (EUCAST), documento EDef 7.1, que ha demostrado ser equivalente al estándar CLSI en levaduras no fermentadoras ${ }^{12-14}$; constituye una metodología alternativa y sus diferencias con el método del CLSI se discutirán más adelante. 
En esta revisión se analizarán los métodos actualmente disponibles para evaluar susceptibilidad antifúngica in vitro, sus ventajas y sus desventajas.

\section{Métodos de dilución en caldo}

Constituyen el estándar de oro para determinar la susceptibilidad in vitro, tanto de levaduras como de hongos filamentosos y miden CIM a distintos fármacos antifúngicos, como anfotericina B, fluocitosina, fluconazol, ketoconazol, itraconazol y los nuevos triazoles como voriconazol, posaconazol y ravuconazol.

En la actualidad se dispone de dos estándares que utilizan el método de microdilución en caldo con este propósito. Uno es el documento M27-A2, de la CLSI, en el cual se han establecido puntos de corte después de una exhaustiva correlación clínica y epidemiológica para definir susceptibilidad in vitro a determinados antifúngicos como los azoles ${ }^{6,8,11}$. Se han determinado puntos de corte de CIM para fluconazol, itraconazol, voriconazol y fluocitosina (Tabla 1$)^{11}$. Los puntos de corte para las equinocandinas (caspofungina, micafungina, anidulafungina) contra Candida, aún no han sido validados; sin embargo, es importante señalar que C. parapsilosis, C. guilliermondii y C. famata tienden a presentar CIMs más altas a estos fármacos ${ }^{4,11}$. El otro es el estándar europeo EUCAST-AFST, cuya versión definitiva se publicó el año 2007 y que ha establecido puntos de corte clínicos de acuerdo a la especie y la relación entre criterios farmacocinéticos y farmacodinámicos y la distribución de las CIMs. Es así como hay especies con puntos de corte relacionados y no relacionados (Tabla 2$)^{14,15}$.

Aunque ambos estándares son similares, presentan diferencias en varios aspectos en la metodología para evaluar susceptibilidad de levaduras fermentadoras como Candida $\mathrm{sp}^{8,11,13,14}$. Algunas de las diferencias más importantes son: el tiempo de incubación (24 hrs para EUCAST y 48 hrs para el estándar CLSI) pues el primero utiliza un inóculo mayor; el fondo del pocillo de la placa de microdilución (plano para EUCAST y redondo para CLSI); la lectura (espectrofotométrica en EUCAST, visual en CLSI). Ambos utilizan RPMI 1640 con glutamina, sin bicarbonato y una concentración de glucosa de 0,2 y $2 \%$ (CLSI y EUCAST, respectivamente) (Tabla 3). Según lo descrito en la literatura científica, ambos estándares correlacionan bastante bien dentro de tres diluciones; sin embargo, el método EUCAST, tendería a leer CIMs más bajas que el método CLSI, siendo la correlación ocasionalmente más baja si con EUCAST se utilizan los puntos de corte de CLSI ${ }^{11}$. Es recomendable leer en cada estándar con sus propios puntos de corte. Desde el punto de vista práctico, el método EUCAST es menos laborioso, más fácil de interpretar, sobre todo en cepas que presentan "trailing" y arroja resultados definitivos a las $24 \mathrm{hrs}$ de incubación. Recientemente

Tabla 1. Puntos de corte, CLSI para Candida, CIM (microdilución en caldo) y diámetro de inhibición (difusión en disco)

\begin{tabular}{|c|c|c|c|c|}
\hline \multirow{2}{*}{$\begin{array}{l}\text { Microdilución } \\
\text { (M27-A) } \\
\text { Antifúngico }\end{array}$} & \multicolumn{4}{|c|}{ CIM $(\mu \mathrm{g} / \mathrm{mL})$} \\
\hline & $\begin{array}{c}\text { Sensible } \\
\text { (S) }\end{array}$ & $\begin{array}{l}\text { Sensible dosis } \\
\text { dependiente } \\
\text { (SDD) }\end{array}$ & $\begin{array}{c}\text { Intermedio } \\
\text { (I) }\end{array}$ & $\begin{array}{c}\text { Resistente } \\
\text { (R) }\end{array}$ \\
\hline Fluconazol & $\leq 8$ & $16-32$ & - & $>64$ \\
\hline Itraconazol & $\leq 0,125$ & $0,25-0,5$ & - & $>1$ \\
\hline Voriconazol & $\leq 1$ & 2 & - & $>4$ \\
\hline Fluocitosina & $\leq 4$ & - & $8-16$ & $>32$ \\
\hline $\begin{array}{l}\text { Difusión en disco } \\
\text { (M-44A) } \\
\text { Antifúngico }\end{array}$ & $\begin{array}{l}\text { Sensible } \\
\text { (S) }\end{array}$ & $\begin{array}{l}\text { Sensible dosis } \\
\text { dependiente } \\
\text { (SDD) }\end{array}$ & $\begin{array}{l}(\mathrm{mm}) \\
\text { Intermedio } \\
\text { (I) }\end{array}$ & $\begin{array}{c}\text { Resistente } \\
\text { (R) }\end{array}$ \\
\hline Fluconazol & $\geq 19$ & $15-18$ & - & $\leq 14$ \\
\hline Voriconazol & $\geq 17$ & $14-16$ & - & $\leq 13$ \\
\hline
\end{tabular}

\begin{tabular}{|c|c|c|c|c|c|c|}
\hline Antifúngico & $\begin{array}{l}\text { Candida } \\
\text { albicans }\end{array}$ & $\begin{array}{l}\quad \text { Espec } \\
\text { Candida } \\
\text { glabrata }\end{array}$ & $\begin{array}{l}\text { untos de c } \\
\text { Candida } \\
\text { krusei }\end{array}$ & $\begin{array}{c}\text { lacionados }(\mathrm{S}< \\
\text { Candida } \\
\text { parapsilosis }\end{array}$ & $\begin{array}{l}\text { Candida } \\
\text { tropicalis }\end{array}$ & $\begin{array}{c}\text { Especies con } \\
\text { puntos de corte } \\
\text { no relacionados } \\
A(S</ R>)\end{array}$ \\
\hline Fluconazol & $2 / 4$ & EI & - & $2 / 4$ & $2 / 4$ & $2 / 4$ \\
\hline \multicolumn{7}{|c|}{$\begin{array}{l}\mathrm{A}=\text { Especies en que los puntos de corte se han establecido en base a la farmacocinética y farmacodinámica del fármaco y no en base a la CIM. B= Cepas con } \\
\text { CIMs superiores a este valor, son raras o aún no se han reportado. Se sugiere repetir la CIM y si se confirma el resultado, enviar la cepa a un laboratorio de } \\
\text { referencia. }-=\text { No se recomienda realizar pruebas de susceptibilidad por tratarse de una especie insensible al fármaco. EI = No hay suficiente evidencia de que } \\
\text { estas especies sean un buen blanco para al fármaco. Ref. } 15\end{array}$} \\
\hline
\end{tabular}




\begin{tabular}{|c|c|c|c|c|c|c|c|}
\hline Método & $\begin{array}{l}\text { Medio de } \\
\text { cultivo }\end{array}$ & $\begin{array}{l}\text { Supl. } \\
\text { glucosa } \\
(\%)\end{array}$ & $\begin{array}{c}\text { Placas de } \\
\text { microdilución }\end{array}$ & $\begin{array}{l}\text { Inoculo } \\
\text { (ufc/ml) }\end{array}$ & $\begin{array}{c}\text { Tiempo de } \\
\text { incubación } \\
\text { (hrs) }\end{array}$ & Lectura & Puntos de corte azoles \\
\hline EUCAST-AFST & $\begin{array}{l}\text { RPMI } 1640 \text { con } \\
\text { glutamina y sin } \\
\text { bicarbonato }\end{array}$ & 2 & $\begin{array}{l}96 \text { pocillos, } \\
\text { fondo plano }\end{array}$ & $\begin{array}{l}0,5 \times 10^{5} \\
2,5 \times 10^{5}\end{array}$ & 24 & espectrofotométrica & $\begin{array}{l}\text { La más baja concentración } \\
\text { que inhiba el crecimiento } \\
\text { en } 50 \% \text { respecto al } \\
\text { control }\end{array}$ \\
\hline CLSI M27-A2 & $\begin{array}{l}\text { RPMI } 1640 \text { con } \\
\text { glutamina y sin } \\
\text { bicarbonato }\end{array}$ & 0,2 & $\begin{array}{c}96 \text { pocillos, } \\
\text { fondo redondo }\end{array}$ & $\begin{array}{l}0,5 \times 10^{3-} \\
2,5 \times 10^{3}\end{array}$ & 48 & visual & $\begin{array}{l}\text { La más baja concentración } \\
\text { que inhiba el crecimiento } \\
\text { sustancialmente comparado } \\
\text { con el control }\end{array}$ \\
\hline
\end{tabular}

se han realizado estudios para validar la lectura de las CIMs de levaduras del género Candida a las $24 \mathrm{hrs}$ utilizando el método del CLSI con buenos resulta$\operatorname{dos}^{16,17}$, aunque se recomienda tener precaución con C. glabrata sensible dosis dependiente (SDD), que podría verse afectada por este cambio $^{16}$.

Para evaluar susceptilidad antifúngica a hongos filamentosos se dispone del documento M38-A del CLSI, estandarizado para Aspergillus sp, Fusarium sp, Rhizopus sp, Pseudalescheria boydii y la forma filamentosa de Sporotrix shenckii. Parte del comité se encuentra trabajando en la estandarización para determinar la CIM de dermatofitos ${ }^{9,18}$. El EUCAST por su parte se encuentra estandarizando la determinación de la CIM para Aspergillus sp ${ }^{11,19,20}$. En general la determinación de la CIM, de hongos fillamentos es más complicada, pues además del recuento por densidad óptica, requiere un recuento de conidios en cámara de Neubauer, debido a la presencia de conidios y elementos hifales que influyen en la preparación del inóculo. Un parámetro que se utiliza en hongos filamentosos, además de la CIM, es la concentración efectiva mínima (CEM), que es la más baja concentración de fármacos capaz de producir un crecimiento hifal aberrante (se expresa en $\mu \mathrm{g} / \mathrm{ml}$ al igual que la CIM). Permite mejorar la interpretación de la CIM de Aspergillus a equinocandinas ${ }^{11}$.

Las desventajas de las pruebas de susceptibilidad basados en microdilución son:

- Necesitan de un largo tiempo para informar los resultados (24-72 hrs).

- Fallan en discriminar cepas resistentes a anfotericina B (el uso de medio AM3 o de E-test ${ }^{\circledR}$ es mejor).

- Aún falta por establecer puntos de corte a varios hongos y fármacos antifúngicos.

- La interpretación de los azoles y fluocitosina es difícil en cepas con "trailing" (Figura 1) 11 .

\section{Método de difusión en disco}

Es un método simple, desarrollado para levaduras y disponible para fármacos solubles en agua, tales como fluocitosina, fluconazol y voriconazol, estandarizado por el CLSI en el documento M44-P para especies de Candida ${ }^{10}$. Se obtiene un halo de inhibición cuya medición correlaciona muy bien con el método de referencia, lo cual ha sido demostrado en estudios multicéntricos que han probado más de 1.000 cepas $^{6}$. La desventaja es que sólo existen puntos de corte para fluconazol y voriconazol (Tabla 1). La utilización de azul de metileno disperso en la superficie de la placa parece mejorar los límites de la zona de inhibición y facilitar la lectura ${ }^{11}$.

\section{Métodos comerciales}

Uno de los más utilizados en los laboratorios de microbiología por su factibilidad de montar y su buena concordancia con los métodos de dilución en caldo es el Etest ${ }^{\circledR}$ (AB BioDisk, Solna, Sweden), aprobado por la Food and Drug Administration (FDA) para susceptibilidad in vitro de Candida sp contra fluconazol e itraconazol $^{7,11}$. Es un método simple, que involucra la inoculación del hongo en la superficie de un agar, seguido de la aplicación de una tira plástica impregnada con un gradiente de concentración del antifúngico, lo cual permite determinar la CIM. Luego, la placa se incuba a $37{ }^{\circ} \mathrm{C}$ por $24-48 \mathrm{hrs}$ y se genera una elipse de inhibición que permite obtener la CIM. Se ha utilizado en levaduras y en hongos filamentosos y mide la CIM a anfotericina B, fluconazol, itraconazol, fluocitosina, voriconazol, posaconazol y caspofungina ${ }^{11}$. Este método ha sido uno de los más eficaces comparado al método de referencia de microdilución para detectar resistencia a anfotericina B en Candida. Se han probado distintos medios siendo el más ulilizado, RPMI suplementado con glucosa al $2 \%$. Otros medios utiliza- 
dos han sido Casitona, para azoles ${ }^{11,21}$, Yeast Nitrogen Base (YNB), particularmente para C. neoformans, AM3 y Mueller-Hinton suplementado con glucosa al 2\% y azul de metileno. Este último medio ha sido especialmente recomendado para discriminar cepas resistentes a anfotericina $\mathrm{B}$, produciendo elipses de inhibición más definidos ${ }^{11}$. Presenta dificultades en la lectura con C. tropicalis, C. neoformans y Trichosporon asahii. Además, tiende a medir CIMs más bajas para voriconazol y más altas para itraconazol en Aspergillus ${ }^{6,11,22}$.

Un sistema de tabletas comerciales (neo-Sensitabs ${ }^{\circledR}$; Rosco Diagnóstika) se encuentra disponible, pero no correlaciona bien con el método de referencia ${ }^{7,11}$. Un estudio reciente evaluó este método en hongos filamentosos con buenos resultados ${ }^{23}$; sin embargo, se requiere de más estudios para validarlo.

El método comercial cuyo formato se asemeja más a la metodología del CLSI es el Sensitre ${ }^{\circledR}$ YeastOne (TREK Diagnostic Systems). Ha sido aprobado por la FDA y se basa en la microdilución en caldo, pero con un sustrato cromogénico para facilitar la interpretación de la CIM. Esta prueba ha incorporado recientemente la determinación de CIM para voriconazol, caspofungina y posaconazol ${ }^{6,11}$.

Actualmente se encuentra disponible en el mercado un método automatizado para determinar la CIM, Vitek $2^{\circledR}$ (Biomerieux), que utiliza una lectura espectrofotométrica, lo cual facilita la lectura de la CIM. Tiene la ventaja de encontrarse acoplado a la identificación de levaduras. Un estudio multicéntrico reveló un alto nivel de reproducibilidad de los resultados y una concordancia de 93,7 a 97,9\% con el método de dilución en caldo. Además, los resultados pueden obtenerse a partir de las 10 hasta las 26 horas de incubación ${ }^{24}$. Este método está disponible en Chile, al igual que E-test ${ }^{\circledR}$.

\section{Otros métodos}

Disponibles para medir susceptibilidad antifúngica están la determinación de concentración fungicida mínima, determinación de curvas de muerte, citometría de flujo y cuantificación de ergosterol, los cuales, debido a su mayor complejidad, se realizan en laboratorios de referencia o altamente especializados en el tema ${ }^{7,11}$.

\section{Recomendaciones para el estudio de susceptibilidad antifúngica}

La realización de estas pruebas tiene indicaciones precisas:

- Efectuar estudios de vigilancia epidemiológica que permitan conocer los perfiles de susceptibilidad y resistencia de cepas clínicas, aisladas principalmente de infecciones invasoras en un país o zona geográfica.
- Determinar el nivel de resistencia frente a nuevos compuestos con actividad antifúngica.

- Predecir la respuesta clínica y optimizar la terapia de pacientes hospitalizados que no responden al tratamiento, que presentan infecciones fúngicas invasoras o en los que se aíslan cepas con alta tasa de resistencia a fármacos antifúngicos $(\mathrm{Ej}$. C. glabrata y fluconazol) ${ }^{11,25,26}$.

Dado que las técnicas de determinación de CIM para hongos en general requieren de personal entrenado, de infraestructura y son de alto costo, no se recomienda la realización de estas pruebas en laboratorios de microbiología de rutina. Es recomendable derivar a centros de referencia.

\section{Importancia de la identificación de levaduras a nivel de especie}

En general las especies fúngicas que frecuentemente producen infecciones en el ser humano, presentan perfiles conocidos de susceptibilidad a los antifúngi$\cos ^{4}$, que orientan al clínico al momento de iniciar terapia, cuando no es posible contar con la CIM. Por ejemplo, hay levaduras intrínsecamente resistentes a azoles como C. krusei, en las que no se deben usar estos fármacos, o que pueden presentar resistencia a anfotericina B como C. lusitaniae. También se ha descrito que $C$. parapsilosis puede presentar sensibilidad intermedia a equinocandinas (Tabla 4). Por otra parte, las distintas especies de Trichosporon presentan diferente susceptibilidad a los antifúngicos, como T. asahii que es más resistente in vitro a anfotericina $\mathrm{B}$ que a los triazoles ${ }^{27}$. En relación a los hongos filamentosos de interés clínico, los Zygomycetes presentan resistencia intrínseca a los azoles, a excepción de posaconazol, mientras que $A$. fumigatus es sensible a triazoles como itraconazol, voriconazol, posaconazol, a equinocandinas y anfotericina B (Tabla 5) ${ }^{4}$.

Los laboratorios de microbiología deben realizar el diagnóstico a nivel de especie, sobre todo en levaduras aisladas del torrente sanguíneo y líquidos estériles.

\section{Interpretación de los resultados}

Un valor de CIM sin una correlación clínica tiene poco valor. En el caso de las infecciones fúngicas, los factores del hospedero juegan un papel muy importante en la respuesta a tratamiento. Por otra parte, la susceptibilidad in vitro no siempre predice el éxito del tratamiento y no siempre la resistencia in vitro se asocia a falla en el tratamiento ${ }^{7}$. Esto ha dificultado el establecimiento de puntos de corte.

En relación a la lectura de la CIM propiamente tal, en algunos de los métodos es visual, dependiendo de la experiencia del operador. Hay cepas productoras de 


\begin{tabular}{|c|c|c|c|c|c|c|}
\hline \multirow[b]{2}{*}{$\begin{array}{l}\text { Especie de } \\
\text { Candida }\end{array}$} & \multirow[b]{2}{*}{ Fluconazol } & \multirow[b]{2}{*}{ Itraconazol } & \multicolumn{2}{|c|}{ Susceptibilidad } & \multirow[b]{2}{*}{ Equinocandinas } & \multirow[b]{2}{*}{ Anfotericina B } \\
\hline & & & Voriconazol & Posaconazol & & \\
\hline C. albicans & $S$ & $S$ & $S$ & $S$ & $S$ & $S$ \\
\hline C. glabrata & $S D D$ a $R$ & $\mathrm{SDD}$ a $\mathrm{R}$ & $\mathrm{SDD}$ a $\mathrm{R}$ & $S$ & S & $\mathrm{S}$ a $\mathrm{I}$ \\
\hline C. parapsilosis & $S$ & $S$ & $S$ & $S$ & S a I & $S$ \\
\hline C. tropicalis & $S$ & $S$ & $S$ & S & S & $S$ \\
\hline C. krusei & $\mathrm{R}$ & $\mathrm{SDD}$ a $\mathrm{R}$ & $\mathrm{S}$ & $S$ & S & S a I \\
\hline C. Iusitaniae & $S$ & S & $\mathrm{s}$ & $S$ & $\mathrm{~S}$ a $\mathrm{I}$ & $S$ a R \\
\hline C. guilliermondii & $S$ & $S$ & $\mathrm{~S}$ & $\mathrm{~S}$ & S & $S$ a $R$ \\
\hline C. dubliniensis & SDD & $S$ & $S$ & $S$ & S & s \\
\hline
\end{tabular}

Tabla 5. Perfil de susceptibilidad de hongos filamentosos de importancia médica

\begin{tabular}{|c|c|c|c|c|c|}
\hline Especie hongo filamentoso & Itraconazol & Voriconazol & $\begin{array}{c}\text { Susceptibilidad } \\
\text { Posaconazol }\end{array}$ & Equinocandinas & Anfotericina B \\
\hline Aspergillus fumigatus & S & S & S & S & S \\
\hline Aspergillus terreus & s & s & s & s & $\mathrm{R}$ \\
\hline Scedosporium apiospermum & S & S & S & R & R \\
\hline Scedosporium prolificans & $\mathrm{R}$ & $\mathrm{R}$ & $\mathrm{R}$ & $\mathrm{R}$ & R \\
\hline Fusarium solanii & $\mathrm{R}$ & $S$ a R & s & $\mathrm{R}$ & s \\
\hline Zygomycetes & R & $\mathrm{R}$ & S & R & s \\
\hline
\end{tabular}

Figura 1. Placa de microdilución con fondo plano (metodología EUCAST-AFST). La columna $\mathrm{n}^{\circ} 11$ y 12 corresponden a control de crecimiento y control negativo respectivamente. Las columnas de la 1 a la 10 corresponden a diluciones decrecientes de fluconazol de 64 a 0,12 $\mu \mathrm{g} /$ $\mathrm{mL}$. Las cepas de las filas B y C presentan "trailing". La lectura de la CIM es espectrofotométrica, a las $24 \mathrm{hrs}$ de incubación. (Gentileza del Laboratorio de Micología Médica, Programa de Microbiología y Micología, ICBM). "trailing" que dificultan la interpretación (Figura 1). Una forma de objetivar la lectura de la CIM es realizar una lectura espectrofotométrica, lo cual recomienda el

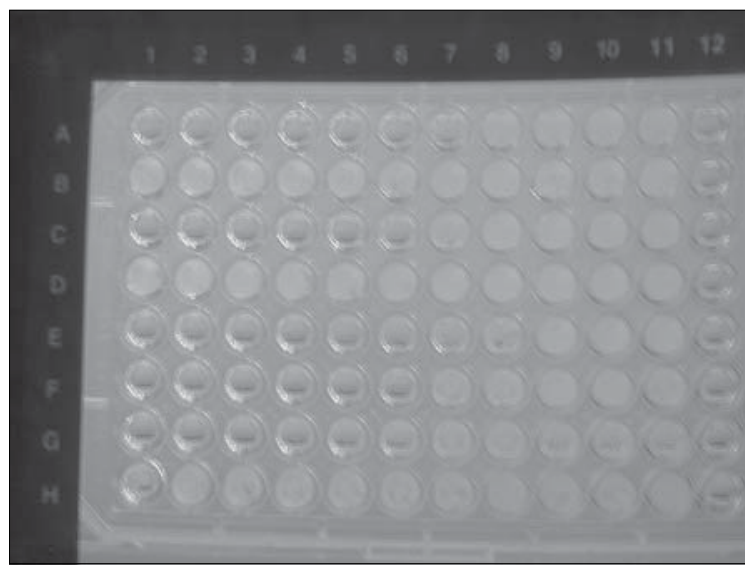

estándar EUCAST ${ }^{11,14}$. El uso de azul de metileno facilita la lectura del halo o elipse de inhibición en los métodos de difusión en agar ${ }^{7,11}$.

El uso de métodos estandarizados permite, en gran medida, disminuir la generación de resultados erróneos; al igual el uso de puntos de corte validados de acuerdo a cada metodología.

\section{Conclusiones}

El desarrollo de métodos estandarizados de susceptibilidad antifúngica, a pesar de las dificultades que presentan, constituyen un notable avance en la terapia de infecciones fúngicas, sobre todo las que comprometen la vida del paciente. Actualmente se cuenta con puntos de corte para varios antifúngicos de uso clínico y para levaduras frecuentemente aisladas de infecciones fúngicas invasoras (p. ej:. Candida, 
Cryptococcus). Aún falta establecer puntos de corte para otras levaduras y hongos filamentosos; sin embargo, con la información de los métodos estandarizados se ha podido detectar cepas intrínsecamente resistentes a los antifúngicos y cepas con CIMs más elevadas que lo habitual, asociado a falla terapéutica.

La pesquisa de cepas con resistencia secundaria ha permitido, además de cambiar y/o ajustar la terapia de los pacientes, identificar mecanismos de resistencia, dando pie a la modificación química de fármacos existentes (p. ej:. voriconazol) y/o a la búsqueda de nuevos fármacos dirigidas a otros blancos de la célula fúngica (p. ej:. equinocandinas).

Es necesario contar con un laboratorio nacional de referencia donde realicen los estudios de vigilancia y las CIMs de las levaduras aisladas de los centros hospitalarios públicos que no cuenten con los recursos humanos y/o económicos para realizar estas pruebas.

Agradecimientos. A Ana María Matamala y Andrea Maccioni por su colaboración en la búsqueda bibliográfica y a Claudio Alburquenque por la lectura crítica del trabajo. Todos son alumnos del Programa de Microbiología y Micología ICBM., Facultad de Medicina de la Universidad de Chile.

\section{Resumen}

Debido al aumento en las infecciones fúngicas invasoras y a la emergencia de hongos resistentes a los antifúngicos, ha sido necesario desarrollar métodos estandarizados de susceptibilidad antifúngica. El Clinical Laboratory Standards Instutute (CLSI) y el European Committee for Antimicrobial Susceptibility Testing (EUCAST) han elaborado guías para susceptibilidad de levaduras por microdilución en caldo (documentos M27-A2 y E. Dis. 7.1, respectivamente). Ambos son equivalentes, aunque presentan diferencias metodológicas y en sus puntos de corte. El CLSI ha desarrollado los documentos M38-A (hongos filamentosos) y M44-A (difusión en disco), mientras que EUCAST trabaja en un documento para Aspergillus sp. Por otra parte, existen métodos comerciales que presentan buena correlación con los métodos de referencia como E-test ${ }^{\circledR}$, Sensititre ${ }^{\circledR}$ y Vitek2 ${ }^{\circledR}$. La interpretación de los resultados debe ser cuidadosa pues la determinación de la concentración inhibitoria mínima (CIM) es muy dificultosa para hongos, hay factores del hospedero involucrados y no siempre hay una correlación entre la CIM y la respuesta a tratamiento. Como estas técnicas, en general, son laboriosas y requieren de personal entrenado, es recomendable derivar los estudios de susceptibilidad a un laboratorio de referencia.

\section{Referencias}

1.- Richardson M, Lass-Flörl C. Changing epidemiology of systemic fungal infections. Clin Microbiol Infect 2008; 14 Suppl 4: 5-24.

2.- Marr K A, Bowden R A. Clinical, cellular, and molecular factors that contribute to antifungal drug resistance. White TC. Clin Microbiol Rev 1998; 11: 382-402.

3.- Ghannoum M A, Rice L B. Antifungal agents: mode of action, mechanisms of resistance, and correlation of these mechanisms with bacterial resistance. Clin Microbiol Rev 1999; 12: 501-17.

4.- Forrest G. Role of antifungal susceptibility testing in patient management. Curr Opin Infect Dis 2006; 19: 538-43.

5.- Gadea I, Cuenca-Estrella M, Martín E, Pemán J, Pontón J, Rodríguez-Tudela J L. Microbiological procedures for diagnosing mycoses and for antifungal susceptibility testing. Enferm Infecc Microbiol Clin 2007; 25: $336-40$

6.- Johnson E M. Issues in antifungal susceptibility testing. J Antimicrob Chemother 2008; 61 Suppl 1: i13-8.
7.- Rex J H, Pfaller M A, Walsh T J, Chaturvedi $\mathrm{V}$, Espinel-Ingroff A, Ghannoum $\mathrm{M}$ A, et al. Antifungal susceptibility testing: practical aspects and current challenges. Clin Microbiol Rev 2001; 14: 643-58.

8.- National Committee for Clinical Laboratory Standards. Reference method for broth dilution antifungal susceptibility testing of yeasts; approved standard NCCLS document M27-A2. Wayne, PA: National Committee for Clinical Laboratory Standard; 2002.

9.- National Committee for Clinical Laboratory Standards. Reference method for broth dilution antifungal susceptibility testing of filamentous fungi; approved standard NCCLS document M38-A. Wayne, PA: National Committee for Clinical Laboratory Standards 2002.

10. - National Committee for Clinical Laboratory Standards. Methods for antifungal disk diffusion susceptibility testing of yeasts: approved standard M44-A. NCCLS, Wayne, PA, USA, 2004.

11.- Arikan S. Current status of antifungal susceptibility testing methods. Med Mycol 2007; 45: 569-87.

12.- Espinel-Ingroff A, Barchiesi F,
Cuenca-Estrella M, Pfaller M A, Rinaldi M, Rodríguez-Tudela J L, et al. International and multicenter comparison of EUCAST and CLSI M27-A2 broth microdilution methods for testing susceptibilities of Candida spp. to fluconazole, itraconazole, posaconazole, and voriconazole. J Clin Microbiol 2005; 43: 3884-9.

13. - Rodriguez-Tudela J L, Donnelly J P, Pfaller M A, Chryssantou E, Warn P, Denning D W, et al. Statistical analyses of correlation between fluconazole MICs for Candida spp. assessed by standard methods set forth by the European Committee on Antimicrobial Susceptibility Testing (E. Dis. 7.1) and CLSI (M27-A2). J Clin Microbiol 2007; 45: 109-11.

14.- Subcommittee on Antifungal Susceptibility Testing (AFST) of the ESCMID European Committee for Antimicrobial Susceptibility Testing (EUCAST). EUCAST definitive document EDef 7.1: method for the determination of broth dilution MICs of antifungal agents for fermentative yeasts. Clin Microbiol Infect 2008; 14: 398-405. 
15.- Antimicrobials for Candida infectionsEUCAST clinical MIC breakpoints 2008-07-24 (v 2.0) http://www.srga.org/ eucastwt/MICTAB/index.html. (accedido 11 noviembre 2008).

16.- Ostrosky-Zeichner L, Rex J H, Pfaller M A, Diekema D J, Alexander B D, Andes D, et al. Rationale for reading fluconazole MICs at 24 hours rather than 48 hours when testing Candida spp. by the CLSI M27-A2 standard method. Antimicrob Agents Chemother 2008; 52: 4175-7.

17.- Pfaller M A, Boyken L B, Hollis R J, Kroeger J, Messer S A, Tendolkar S, et al. Validation of 24-hour fluconazole MIC readings versus the CLSI 48-hour broth microdilution reference method: results from a global Candida antifungal surveillance program. J Clin Microbiol 2008; 46: 3585 90 .

18.- Ghannoum M A, Arthington-Skaggs B, Chaturvedi V, Espinel-Ingroff A, Pfaller M A, Rennie R, et al. Interlaboratory study of quality control isolates for a broth microdilution method (modified CLSI M38A) for testing susceptibilities of dermatophytes to antifungals. J Clin Microbiol 2006; 44: 4353-6.
19.- Chryssanthou E, Cuenca-Estrella M. Comparison of the EUCAST-AFST broth dilution method with the CLSI reference broth dilution method (M38-A) for susceptibility testing of posaconazole and voriconazole against Aspergillus spp. Clin Microbiol Infect 2006; 12: 901-4.

20.- Lass-Flörl C, Perkhofer In vitro susceptibility-testing in Aspergillus species. S Mycoses 2008; 51: 437-46.

21.- Tapia C, León E, Palavecino E. Antifungal susceptibility of yeasts by Etest. Comparison of 3 media Rev Med Chile 2003; 131: 299302.

22.- Pfaller J B, Messer S A, Hollis R J, Diekema D J, Pfaller M A. In vitro susceptibility testing of Aspergillus spp: comparison of Etest and reference microdilution methods for determining voriconazole and itraconazole MICs. J Clin Microbiol 2003; 41: 1126-9.

23.- Espinel-Ingroff A, Canton E. Comparison of Neo-Sensitabs tablet diffusion assay with CLSI broth microdilution M38-A and disk diffusion methods for testing susceptibility of filamentous fungi with amphotericin B, caspofungin, itraconazole, posaconazole, and voriconazole. J Clin Microbiol 2008; 46: 1793-803.

24.- Pfaller M A, Diekema D J, Procop G W, Rinaldi M G. Multicenter comparison of the VITEK 2 antifungal susceptibility test with the CLSI broth microdilution reference method for testing amphotericin B, flucytosine, and voriconazole against Candida spp. J Clin Microbiol 2007; 45: 3522-8.

25.- Cuenca-Estrella M, Rodríguez-Tudela J L. Should antifungal treatments be based upon results of antifungal susceptibility testing? Rev Iberoam Micol 2002; 19: 133-8.

26.- Gadea I, Cuenca-Estrella M. Infección fúngica invasora (MICOMED); Estudio de la infección en el trasplante (GESITRA); Sociedad Española de Enfermedades Infecciosas y Microbiología Clínica (SIMC). Guidelines for fungal diagnoses and antifungal sensitivity studies. Enferm Infecc Microbiol Clin 2004; 22: 32-9.

27.- Rodríguez-Tudela J L, Díaz-Guerra T M, Mellado E, Cano V, Tapia C, Perkins A, et al. Susceptibility patterns and molecular identification of Trichosporon species. Antimicrob Agents Chemother 2005; 49: 4026-34. 